\title{
NBS
}

Eechnical Note No.200

CALCULATION OF SUNRISE AND SUNSET TIMES AT IONOSPHERIC HEIGHTS ALONG A GREAT CIRCLE PATH

A. H. Brady and D. D. Crombie

U. S. DEPARTMENT OF COMMERCE NATIONAL BUREAU OF STANDARDS 


\section{THE NATIONAL BUREAU OF STANDARDS}

The National Bureau of Standards is a principal focal point in the Federal Government for assuring maximum application of the physical and engineering sciences to the advancement of technology in industry and commerce. Its responsibilities include development and maintenance of the national standards of measurement, and the provisions of means for making measurements consistent with those standards; determination of physical constants and properties of materials; development of methods for testing materials, mechanisms, and structures, and making such tests as may be necessary, particularly for government agencies; cooperation in the establishment of standard practices for incorporation in codes and specifications; advisory service to government agencies on scientific and technical problems; invention and development of devices to serve special needs of the Government; assistance to industry, business, and consumers in the development and acceptance of commercial standards and simplified trade practice recommendations; administration of programs in cooperation with United States business groups and standards organizations for the development of international standards of practice; and maintenance of a clearinghouse for the collection and dissemination of scientific, technical, and engineering information. The scope of the Bureau's activities is suggested in the following listing of its four Institutes and their organizational units.

Institute for Basic Standards. Electricity. Metrology. Heat. Radiation Physics. Mechanics. Applied Mathematics. Atomic Physics. Physical Chemistry. Laboratory Astrophysics.* Radio Standards Laboratory: Radio Standards Physics; Radio Standards Engineering.** Office of Standard Reference Data.

Institute for Materials Research. Analytical Chemistry. Polymers. Metallurgy. Inorganic Materials. Reactor Radiations. Cryogenics. * Office of Standard Reference Materials.

Central Radio Propagation Laboratory.** Ionosphere Research and Propagation. Troposphere and Space Telecommunications. Radio Systems. Upper Atmosphere and Space Physics.

Institute for Applied Technology. Textiles and Apparel Technology Center. Building Research. Industrial Equipment. Information Technology. Performance Test Development. Instrumentation. Transport Systems. Office of Technical Services. Office of Weights and Measures. Office of Engineering Standards. Office of Industrial Services.

* NBS Group, Joint Institute for Laboratory Astrophysics at the University of Colorado.

** Located at Boulder, Colorado. 


\title{
NATIONAL BUREAU OF STANDARDS Eechnical Note 209 \\ Issued November 8, 1964
}

\section{CALCULATION OF SUNRISE AND SUNSET TIMES AT IONOSPHERIC HEIGHTS ALONG A GREAT CIRCLE PATH}

\author{
A. H. Brady and D. D. Crombie \\ Central Radio Propagation Laboratory \\ National Bureau of Standards \\ Boulder, Colorado
}

NBS Technical Notes are designed to supplement the Bureau's regular publications program. They provide a means for making available scientific data that are of transient or limited interest. Technical Notes may be listed or referred to in the open literature.

For sale by the Superintendent of Documents, U. S. Government Printing Office Washington, D.C. 20402

Price: 20 cents 



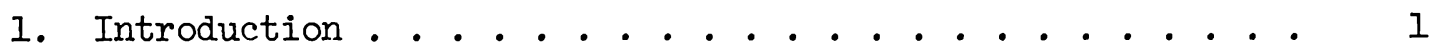

2. Determination of Sunrise and Sunset Times . . . . . 2

3. Determination of Coordinates of Points Along the Path. . 4

4. A Digital Computer Program to Determine the Amount of Path Illumination. . . . . . . . . . . . . . . . . .

4.1 Form of Computer Output . . . . . . . . . . 5

4.2 The Computer Program. . . . . . . . . . . 6

5. Some Comparisons of Path Illumination with Diurnal Variations of VLF Phase. . . . . . . . . . . . . . . . 7

6. Discussion . . . . . . . . . . . . . . 8

7. Acknowledgment . . . . . . . . . . . . . . 9

8. References . . . . . . . . . . . . . . . 10

Figures. . . . . . . . . . . . . . . . . . . . 11 

Calculation of Sunrise and Sunset Times at Ionospheric

Heights along a Great Circle Path

A. H. Brady and D. D. Crombie

Formulae for determining

the coordinates of points along

a specified great circle path

and for determining the times of

sunset and sunrise at these points

are given. A computer program for

making the calculations and showing

which portions of a great circle

path are illuminated is outlined.

Some illustrations of the dependence

of VLF phase delay on path illumina-

tion are also given.

\section{Introduction}

There is, at present, considerable interest in the diurnal variation in the phase of a VLF signal at great distances and in its dependence upon the distribution of daylight and darkness along the propagation path [Crombie et al., 1958]. For this reason, a simple and straightforward numerical procedure has been devised for the computation of the times of sunrise and sunset at a specified height along a great circle path. The computation then yields the fraction of the path which is illuminated at any time. It is the authors' experience that much time can be wasted both in deciding which spherical trigonometrical formulae should be used and in programming a computer to make the calculations correctly. Thus, although this report contains nothing mathematically new or sophisticated, it is hoped that the collection of formulae and the outline of the computer program may be useful to others who may have to make such calculations for the first time, or have large numbers of such calculations to make.

Following a discussion of how sunrise or sunset is defined above the surface of the earth, expressions will be given for calculating the time at which the sun reaches a given zenith angle, for a specified longitude and latitude and specified declination of the sun. In section 3, formulae are given for finding the latitude and longitude of $\mathrm{n}+\mathrm{I}$ uniformly spaced points along a great circle path (less than $180^{\circ}$ long) whose end points are specified. These values can then be inserted in the earlier formulae and the sunset and sunrise times obtained. Section 4 discusses the problem of using a computer for making these calculations. Section 5 gives some examples of the calculations and compares them with observed diurnal phase variations. Accuracy adequate for the intended purpose is obtained with a considerable simplification by assuming that (a) the sun can be treated as a point source, (b) 
atmospheric refraction can be neglected, and (c) the earth is a sphere. Some consequences of the resulting approximations are discussed in section 6 .

\section{Determination of Sunrise and Sunset Times}

Sunrise or sunset is usually thought of as occurring when the sun rises or sets, respectively, above or below the horizon, and the time at which this occurs depends upon the elevation of the observer above the surrounding country. At a point on the surface of a smooth, spherical, airless earth, the sun's rays would be horizontal when it sank below the horizon at sunset; i.e., its zenith angle $x$ would be $90^{\circ}$. For points above the surface, the zenith angle at sunrise or sunset increases as the distance of the observer above the surface increases.

In speaking of sunrise or sunset in the ionosphere, we are of course interested in those components of the sun's radiation which affect the ionization, and most of these cannot penetrate the lower atmosphere. For example, the ozone layer screens the near ultra-violet light from heights below about $30 \mathrm{~km}$ [Mitra, 1952; Reid, 1961]. In general, therefore, at a given point above the screening height (S) for a particular wavelength, sunrise and sunset for this wavelength occur when the sun's rays, arriving at the point, are horizontal at the screening point. From the geometry illustrated in figure l, it is easily shown that the value of $x$ at a height $H$, at which the sun's rays become tangential to a concentric sphere of radius $A+S$, is given by

$$
\cos x=-\sqrt{1-\left(\frac{A+S}{A+H}\right)^{2}}
$$

Since it is mainly values of $x$ between $90^{\circ}$ and $100^{\circ}$ that are of interest, and, since $\mathrm{A}$ is much greater than both $\mathrm{S}$ and $\mathrm{H}$, equation $I$ can be reduced to the approximate form

$$
x \approx 90^{\circ}+1.01 \sqrt{\mathrm{H}-\mathrm{S}}
$$

which is useful for hand calculations. This gives $x$ in degrees when $H$ and $S$ are expressed in kilometers. A plot of this relation is given in figure 1 along with the sunrise-sunset geometry.

Equation 1 shows that there is no unique combination of $\mathrm{S}$ and $\mathrm{H}$ which will yield a particular value of $\chi$ (when $H>0$ ). Thus rather than using particular values of $H$ and $S$ it is better, in general, to use the appropriate value of $x$. Nevertheless it is sometimes convenient to quote the value of $\mathrm{H}$ which corresponds, for a particular $\mathrm{S}$, to a given value of $x$. Where this is done here, the screening height, $S=30 \mathrm{~km}$, has been used. 
With $x$ fixed by $\mathrm{H}$ and $\mathrm{S}$, application of the law of cosines to the spherical triangle whose vertices are the north pole, the sun, and the zenith gives the local hour angle (IHA) at sunset and sunrise in the following form [Mitra, 1952]:

$$
\cos (\mathrm{LHA})=\frac{\cos x-\sin \varphi_{D} \sin \delta}{\cos \varphi_{D} \cos \delta}
$$

where $\varphi_{D}$ is the latitude of the point on the earth and $\delta$ is the declination of the sun at noon on the day in question ( 1 ). (North declinations and latitudes are positive; South declinations and latitudes are negative). The local hour angle of the sun is defined as the angular distance from the meridian of the point in question to the meridian of the sub-solar point.

If $\mid \cos$ LHA $\mid>1$, LHA is imaginary and sunrise and sunset do not occur at the point under consideration. In such a case, account must be taken of the local season to determine whether the point is in darkness or sunlight.

To obtain the Greenwich Mean Time (or Universal Time) for sunrise and sunset, it is necessary to include the Equation of time, $\mathrm{E}$. This is not printed in the Air Almanac but can be calculated from the value of the Greenwich hour angle of the sun at Greenwich mean noon, which is given in the Almanac. (Alternatively, $\mathrm{E}$ can be obtained from the American Ephemeris.) Thus,

$$
\mathrm{E}=-\frac{\mathrm{GHA}}{15^{\circ}} \text { hours } \quad \text { if } \mathrm{GHA}<180^{\circ}
$$

and

$$
\mathrm{E}=\frac{360^{\circ}-\mathrm{GHA}}{15^{\circ}} \text { hours } \quad \text { if } \mathrm{GHA}>180^{\circ} \text {. }
$$

where $\quad E=$ mean time - apparent time.

The Universal Time of sunrise (TR) and sunset (TS) in hours are then given by,

Footnote (1). Use of the value of declination at noon, instead of at sunrise (or sunset) may introduce small errors in the calculated time of sunrise (or sunset) at very high latitudes near the equinoxes. These errors may be reduced by using an iterative process. 


$$
\left.\begin{array}{l}
\mathrm{TS} \\
\mathrm{TR}
\end{array}\right\}=12+\mathrm{E}-\theta_{\mathrm{D}} \text { (In hours) } \pm \mathrm{LHA} \text { (In hours) }
$$

where $\theta_{D}$ is the longitude of the point expressed in hours (taking East to be positive and West to be negative), and the algebraic sign of LHA is chosen to correspond to sunrise (-) or sunset (+). Equation 3 may yield values for TR and TS which are either negative or greater than 24 hours. To avoid these difficulties, it is suggested that one simply add 24 hours to the result as a matter of routine, and then compute this result (modulo 24) to obtain proper clock values for TR and TS.

\section{Determination of Coordinates of Points Along the Path}

To determine the illumination status along the path it is necessary to have the values of the geographic coordinates at any distance $D$ along the path. Figure 2 shows the path coordinate geometry as used in this determination, with $\mathrm{N}$ corresponding to the north pole, $\mathrm{E}$ to the east end of the path, and $W$ to the west end. $\theta_{E}$ and $\theta_{W}$ are the respective longitudes, and $\varphi_{E}$ and $\varphi_{W}$ are the respective latitudes. It is assumed that the earth is spherical.

First, the arc length EW of the path is computed using the law of cosines for a spherical triangle:

$$
\cos E \mathrm{~W}=\sin \varphi_{\mathrm{W}} \sin \varphi_{\mathrm{E}}+\cos \varphi_{\mathrm{W}} \cos \varphi_{\mathrm{E}} \cos \left(\theta_{\mathrm{E}}-\theta_{\mathrm{W}}\right) \cdot
$$

Also, again using the law of cosines, the angle $\mathrm{E}$ is determined:

$$
\cos E=\frac{\sin \varphi_{W}-\sin \varphi_{E} \cdot \cos \overline{E W}}{\cos \varphi_{E} \cdot \sin \overline{E W}}
$$

The path $(\overline{\mathrm{EW}})$ is divided into $\mathrm{n}$ segments so that the angular distance to the end of the $\mathrm{k}^{\text {th }}$ segment from the east end is

$$
\overline{E D}=k \cdot \frac{\overline{E W}}{n} \text {. }
$$

Again the law of cosines is used to determine the latitude $\varphi_{D}$, 
$\sin \varphi_{D}=\cos \overline{E D} \cdot \sin \varphi_{E}+\sin \overline{E D} \cdot \cos \varphi_{E} \cdot \cos E \cdot$

Since $-90^{\circ} \leq \varphi_{D} \leq 90^{\circ}, \cos \varphi_{D}$ will simply be given by $\sqrt{1-\sin ^{2} \varphi_{D}}$.

The longitude $\theta_{D}$ is also determined by the law of cosines. Thus,

$$
\cos \left(\theta_{E}-\theta_{D}\right)=\frac{\cos E D-\sin \varphi_{D} \cdot \sin \varphi_{E}}{\cos \varphi_{D} \cdot \cos \varphi_{E}},
$$

and

$$
\theta_{D}=\theta_{E}-\operatorname{Arccos}\left[\cos \left(\theta_{E}-\theta_{D}\right)\right]
$$

where the principal value of the arccosine has been designated, since $\left(\theta_{E}-\theta_{D}\right)<180^{\circ}$.

$$
\begin{aligned}
& \text { 4. A Digital Computer Program to Determine } \\
& \text { the amount of Path Illumination } \\
& 4.1 \text { Form of Computer Output }
\end{aligned}
$$

In the calculations, using a digital computer, it was found convenient to print out the illumination status of each point on the path graphically, as a function of the time of day. Before outlining the computer program, a brief account will be given of the form of the display.

Figure 3 shows a sample of the output for June 21 on the Rugby (England) to Boulder (Colo.) path at an altitude of $70 \mathrm{~km}$ and for a screening height of $30 \mathrm{~km}$.

The illumination status of each point on the path was calculated at 15 minute intervals, and time is shown in the left-hand column. The path was divided into 100 segments, numbered from East to West along the path and left to right across the top of the page. If a segment is dark, it is shown by "*". The percentage of the length of path illumination is listed down the right-hand edge corresponding to the universal time opposite. It was computed by dividing the number of illuminated segments by the total number of segments.

At the bottom of the figure, the actual sunrise and sunset times are listed for each segment. This is done to furnish more detailed information if it is required. A sunrise occurring at 0.00 hours with sunset at 24.00 hours is used to indicate that the segment is always illuminated. 
(The sun does not set:) A sunset at 0.00 hours with sunrise at 24.00 hours would be used to indicate a segment which remains in darkness. (The sun does not rise!)

Figure 3 shows illumination variations which may occur on relatively high latitude paths at ionospheric heights during summer. During the period when the path, at ground level, is in darkness, there is a short period during which the ionosphere is illuminated by sunlight from over the pole. Figure 3 shows that this occurs, on the Boulder-Rugby path, at approximately 0300 UT on June 2l, and that at this time the whole path is sunlit at ionospheric heights.

\subsection{The Computer Program}

The following input data are necessary for the computer program:

1. Solar declination and Greenwich hour angle at $12^{\mathrm{h}}$ UT for each day for which the calculation is to be made (from Air Almanac).

2. Screening height and height of ionosphere in kilometers.

3. Coordinates of the eastern and western ends of the path.

4. Number ( $n$ ) of segments into which the path is to be divided. (The practical limitation on this number is determined by the number of characters across a page of printed output from the digital computer to be used.)

The program then proceeds in the following steps:

1. Read in the declination $(\delta)$ and Greenwich hour angle (GHA) for each day, convert to radians, and store.

2. Read and store the screening height (S) and ionospheric height (H) in kilometers.

3. Read the coordinates $\left(\varphi_{E}, \theta_{E}, \varphi_{W}, \theta_{W}\right)$ of the end points of the path to be computed and the number of segments ( $n$ ) into which it is to be divided.

4. Convert the coordinates from degrees to radian values. At this point one may make whatever checks on the coordinates are necessary, such as for antipodal end points. The case where the path lies on a meridian should be treated separately to avoid difficulties with "round-off" error in the arguments of the inverse trigonometric functions used in the calculations. (For example, a cosine might become 1.000001 when computed from a formula.) 
5. Compute the angular distance (EW) between path end points, using equation 4. Compute and store the values of sin EW and cos EW.

6. Compute $\cos$ E from equation 5 .

7. For each of the $n+1$ points along the path compute and store $\theta_{D}, \sin \varphi_{D}$, and $\cos \varphi_{D}$ using equations $6,7,8$, and 10.

8. Compute the sunrise and sunset times for the given height at each point along the path, and store these times in an array.

a) Compute cos LHA from equation 2 .

b) If $\mid \cos$ LHA $\mid>1$, sunrise and sunset do not occur. There, if $\left(\delta \cdot \varphi_{D}\right)<0$, it is winter and the point is dark and so set the time of sunrise $=24.00$ hours and sunset $=0.00$ hours and proceed to the next segment. If $\left(\delta . \varphi_{D}\right)>0$, it is summer and the point is always sunlit and so set the time of sunrise $=0.00$ hours and sunset $=24.00$ hours and proceed to the next segment.

c) If $\mid \cos$ LHA $\mid \leq 1$, then compute LHA and convert to hours. Compute the times of sunrise and sunset from equation 3. Add 24 hours to each value and compute the results (Modulo 24). This gives positive values for the times of sunrise and sunset.

9. Determine number of sunlit segments and divide by $n$. Then print a line with the status of each segment represented by the appropriate symbol. Repeat until the time 24- $\Delta t$ is reached.

10. Print out the sunrise and sunset times from 8 , and then repeat steps 8 and 9 for each day and (or) layer height desired. (The new layer heights may be incremented values of the original value of $\mathrm{H}_{\text {.) }}$

5. Some Comparisons of Path Illumination with Diurnal Variations of VLF Phase

The phase of a frequency-stabilized very low frequency (VLF) signal shows a diurnal variation having a nearly trapezoidal shape for paths at temperate or lower latitudes. Pierce [1955] and Crombie et al. [1958] have previously pointed out the dependence of the major features of this variation upon the times of sunrise and sunset at the transmitter and receiver ends of the path. 
An example illustrating this dependence is given in figure 4 which shows approximately the variation with time of the length of path in darkness at $80 \mathrm{~km},\left(x=97^{\circ}\right)$ and at the ground level (solid lines) during September and December 1962 for the path between the Canal Zone and Frankfurt, Germany. The corresponding phase variation for the $18 \mathrm{kc} / \mathrm{s}$ signal propagating from the Canal Zone to Frankfurt are shown by the dashed lines. The correspondence between the phase and illumination curves is quite marked.

At high latitudes the diurnal phase delay may show variations which are not shown by the diurnal variation in the length of the illuminated path at ground level. Such a situation is shown in figure 5 for the GBR (Rugby, England) to Boulder, Colo., path during June 1962 . Again the solid lines (obtained in part from figure 3 ) show the variation in the length of path in darkness at ground level, $70 \mathrm{~km}$ and at $90 \mathrm{~km} \quad(x=0$, $96.5^{\circ}$ and $97.5^{\circ}$ ) approximately. The dashed line is the mean phase delay for the same period. Of particular interest is the dip in the phase curve at $0300 \mathrm{UT}$, which agrees with the increase in illumination shown at the higher levels in the atmosphere, but not at ground level.

\section{Discussion}

Some simplifications of the actual problem have been made here in order to keep the computation simple and because a more sophisticated treatment appeared unnecessary for the work with which these calculations was associated. These simplifications are:

a) the sun can be treated as a point source,

b) atmospheric refraction is neglected,

c) the earth is spherical.

For some purposes it may be necessary to consider the effect of these simplifications. Often, for example, [Mitra,1952] the fact that the sun is not a point source can be included by increasing the angle of $X$ given by equation ( 1 ) and used in equation (2) by 16', the apparent semidiameter of the sun. In the present context this would be tantamount to assuming that full illumination occurred while any portion of the sun is visible and is not necessarily a better assumption than that used in this paper. Again, although screening by the atmosphere has been considered, vertical refraction by the atmosphere has not. At the surface of the earth and for visible light refraction can be included by further increasing the value of $x$ used in equation (2) by 34'. In both these cases neither the effective diameter of the sun nor the correction for refraction is known exactly. In fact it is hoped that the experimental observations may eventually lead to a better understanding of these values.

It should be pointed out, however, that the errors resulting from neglect of these quantities, or from the use of incorrect values for 
them may be appreciable at high latitudes during winter. Since sunrise and sunset in this paper are defined as the times at which the center of the sun passes a given zenith angle these times may be considerably in error when the zenith distance of the sun is changing slowly and is near $90^{\circ}$. For example, neglect of refraction and the sun's diameter at certain places near the Arctic Circle leads to the result that the sun should not rise during mid-winter whereas in fact several hours of sunshine each day are experienced. It should be noted that this is entirely due to the error of about $50^{\prime}$ in determining the zenith distance of the sun and from neglect of states intermediate between full daylight and darkness. It is possible that errors of this type decrease as the screening height increases [Reid, 1961]. Indeed, as noted above, it is expected that comparison of the length of the path illuminated and the corresponding phase change may yield more information on this subject.

The final simplifying assumption listed above is that the earth is spherical. This is only approximately true since the polar and equatorial radii differ by some $22 \mathrm{~km}$. This source of error could be overcome if necessary by using the geocentric latitude (the angle subtended at the earth's center by the arc between the place in question and the equator) instead of the geographic latitude (the angle between the normal to the earth's surface at the place in question and the equatorial plane). It can be shown [Barlow and Bryan, 1944] that the difference between these angles has a maximum value at a latitude of $45^{\circ}$ and that the difference is only about 12'. Errors resulting from this cause have been neglected since at a latitude of $45^{\circ}$ the time of ground sunrise or sunset typically changes at a rate of about 3 minutes for each $1^{\circ}$ change in latitude.

\section{Acknowledgment}

Valuable discussion with J. H. Crary and advice by H. H. Howe on the preparation of this paper are gratefully acknowledged. The work was done as a part of a research program supported by the AdvancedResearch Projects Agency, Washington, D. C., under Order 183. 


\section{References}

Barlow, C. W. C., and G. H. Bryan (1944), Elementary Mathematical Astronomy, 5th Edition, 80 (University Tutorial Press Ltd., Iondon).

Crombie, D. D., A. H. Allan, and M. Newman (May 1958), Phase variations of the $16 \mathrm{kc} / \mathrm{s}$ transmission from Rugby as received in New Zealand, Proc. IEE, Part B, 301-304.

Mitra, S. K. (1952), The upper atmosphere (The Asiatic Society, Calcutta, 2nd Edition).

Pierce, J.A. (1955), The diurnal carrier phase variations of a $16 \mathrm{kc} / \mathrm{s}$ transatlantic signal, Proc. IRE 43, 584-588.

Reid, G. C. (1961), A study of the enhanced ionization produced by solar protons during a polar cap absorption event, J. Geophys. Res. $66,4071-4085$. 
SUNRISE/SUNSET ANGLE OF INCIDENCE VERSUS LAYER HEIGHT FOR DIFFERENT SCREEN HEIGHTS

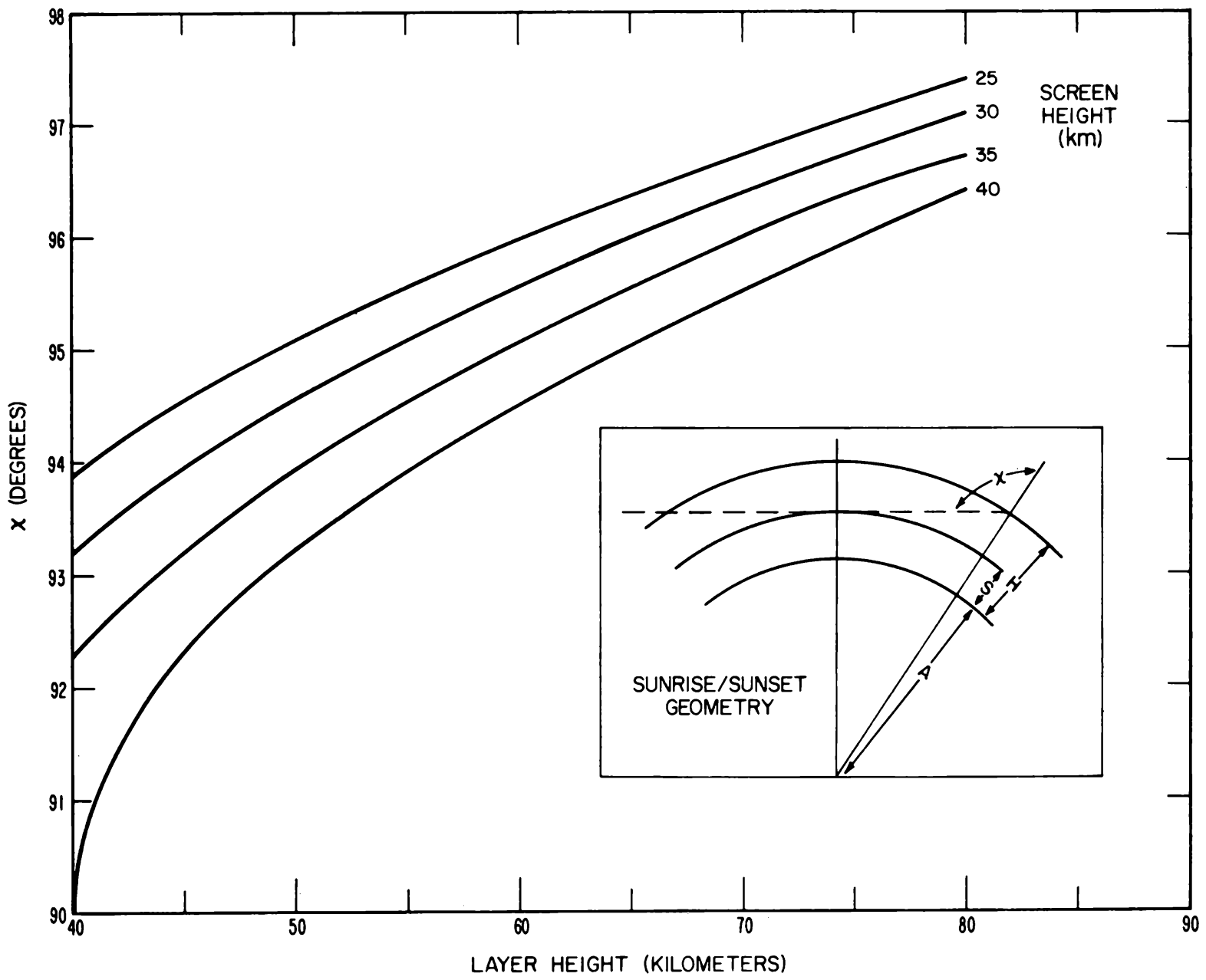

Figure 1. Zenith angle $(X)$ of sun at sunset and sunrise for different altitudes $(\mathrm{H})$, and screening heights $(\mathrm{S})$. The radius of the earth is A. (The inset shows the geometry at sunrise or sunset. The three circular arcs indicate the surface of the earth, the top of the screening layer, and the ionosphere. The sun is assumed to be at the left of the dotted line, ) 
PROPAGATION PATH GEOMETRY

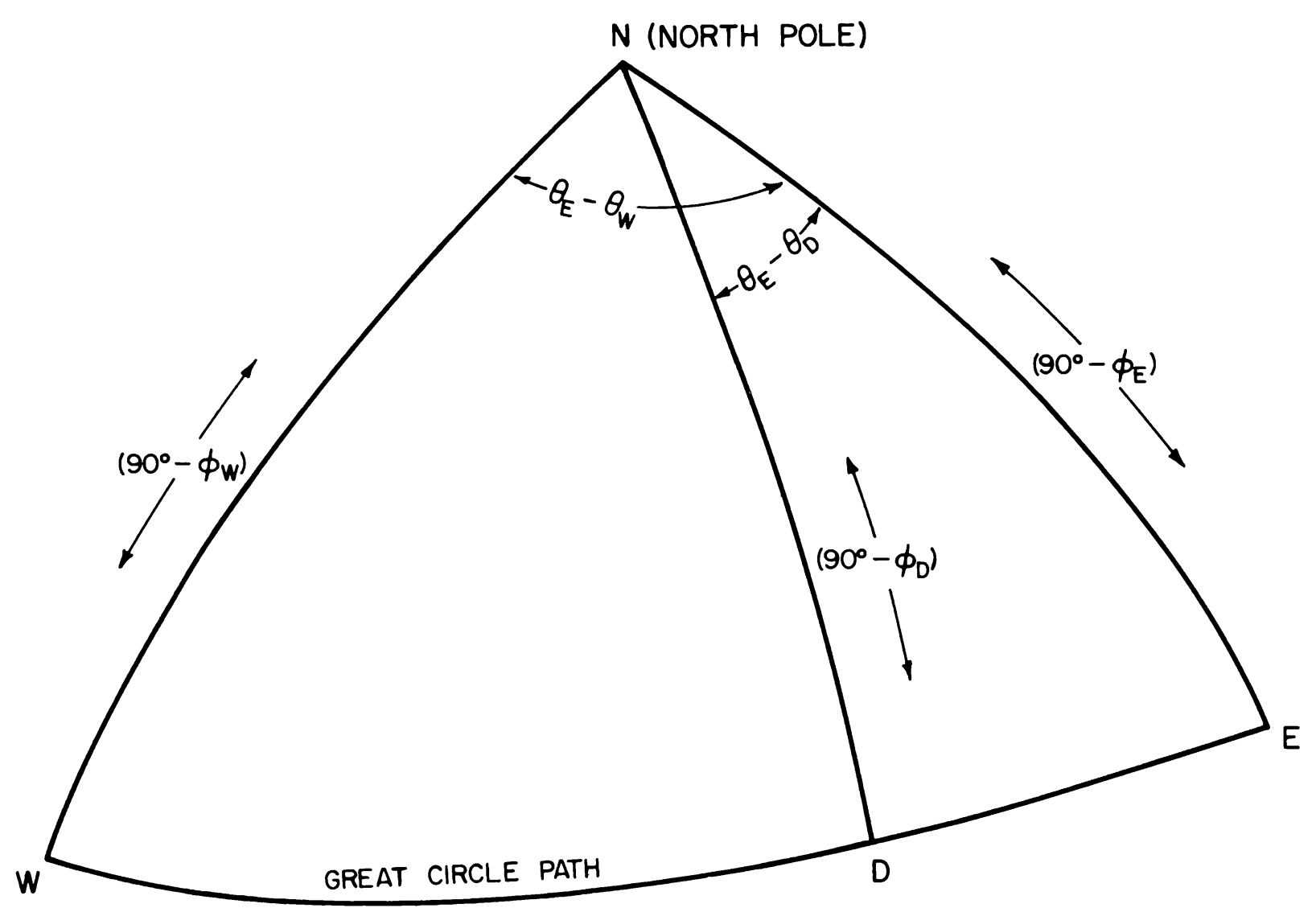

Figure 2. Propagation path geometry. 
DATE 2106 (DAY,MO.), SCREENING HEIGHT $=30$. KM., IONOSPHERIC MEIGHT $=70$. KM., END POINT COORdinates-

LONG. -1.2 DEG, LAT. 52.4 DEG AND LONG.-105.2 DEG, LAT. 40.0 DEg. PATH LENGTH = 7427. KM DIVIDED INTO 100 SEgMENTS.

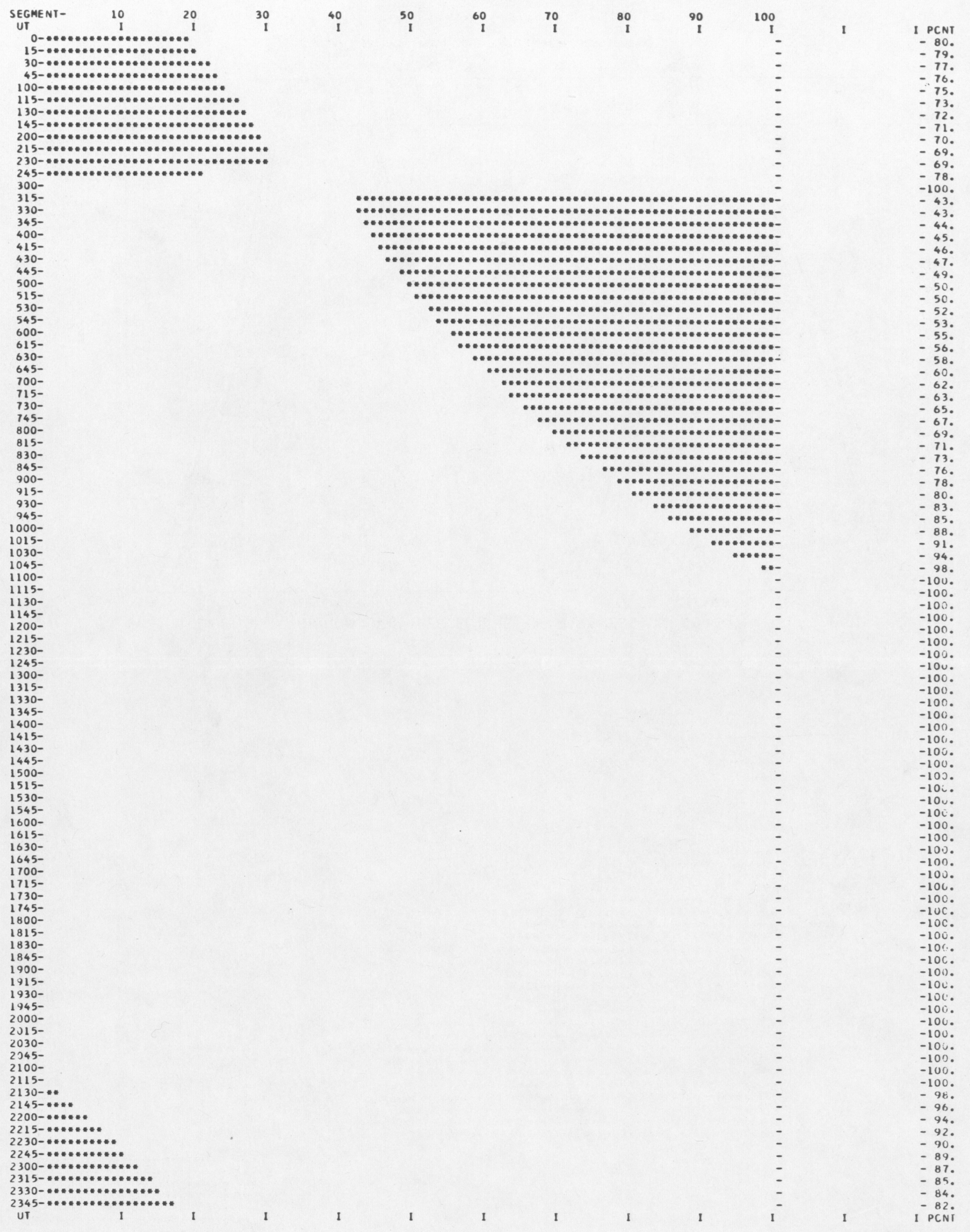

- = DARKNESS

SOlar illumination of the lower ionosphere

over a great circle path

(SEE LEGEND AT TOP)

SUNRI SE AND SUNSET TIMES (HOURS. HOURS) along PATH. DistanCE in Kilometers.

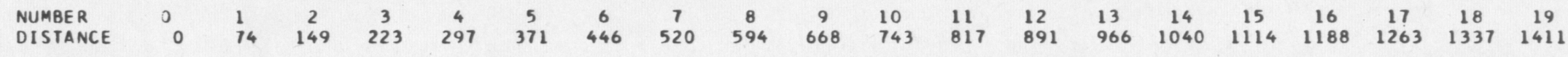
$\begin{array}{llllllllllllllllllllll}\text { SUNRISE } & 2.75 & 2.75 & 2.75 & 2.76 & 2.76 & 2.76 & 2.76 & 2.76 & 2.76 & 2.76 & 2.76 & 2.76 & 2.76 & 2.76 & 2.76 & 2.76 & 2.76 & 2.76 & 2.76 & 2.76\end{array}$

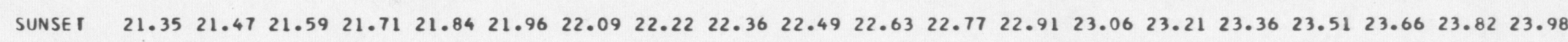
$\begin{array}{lcccccccccccccccccccc}\text { NUMBER } & 20 & 21 & 22 & 23 & 24 & 25 & 26 & 27 & 28 & 29 & 30 & 31 & 32 & 33 & 34 & 35 & 36 & 37 & 38 & 39 \\ \text { DISTANCE } & 1485 & 1560 & 1634 & 1708 & 1782 & 1857 & 1931 & 2005 & 2080 & 2154 & 2228 & 2302 & 2377 & 2451 & 2525 & 2599 & 2674 & 2748 & 2822 & 2897\end{array}$

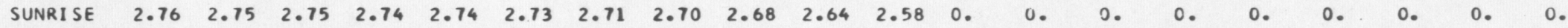

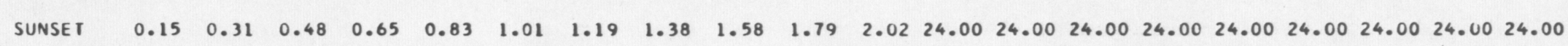
$\begin{array}{lcccccccccccccccccccc}\text { NUMBER } & 40 & 41 & 42 & 43 & 44 & 45 & 46 & 47 & 48 & 49 & 50 & 51 & 52 & 53 & 54 & 55 & 56 & 57 & 58 & 59 \\ \text { DISTANCE } & 2971 & 3045 & 3119 & 3194 & 3268 & 3342 & 3416 & 3491 & 3565 & 3639 & 3714 & 3788 & 3862 & 3936 & 4011 & 4085 & 4159 & 4233 & 4308 & 4382\end{array}$

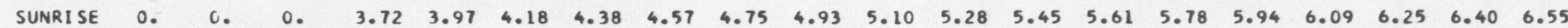

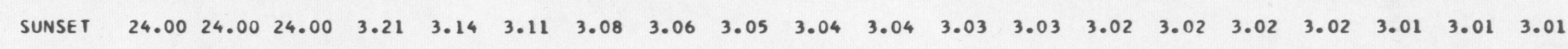
$\begin{array}{lllllllllllllllllllll}\text { NUMBER } & 60 & 61 & 62 & 63 & 64 & 65 & 66 & 67 & 68 & 69 & 70 & 71 & 72 & 73 & 74 & 75 & 76 & 77 & 78 & 79 \\ \text { DISTANCE } & 4456 & 4530 & 4605 & 4679 & 4753 & 4828 & 4902 & 4976 & 5050 & 5125 & 5199 & 5273 & 5347 & 5422 & 5496 & 5570 & 5645 & 5719 & 5793 & 5867\end{array}$ $\begin{array}{llllllllllllllllllllllll}\text { SUNRISE } & 6.70 & 6.85 & 6.99 & 7.13 & 7.27 & 7.41 & 7.54 & 7.67 & 7.80 & 7.93 & 8.05 & 8.17 & 8.29 & 8.41 & 8.53 & 8.64 & 8.75 & 8.86 & 8.97 & 9.07\end{array}$

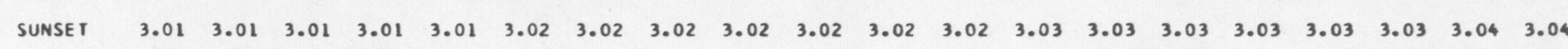
$\begin{array}{lcccccccccccccccccccc}\text { NUMBER } & 80 & 81 & 82 & 83 & 84 & 85 & 86 & 87 & 88 & 89 & 90 & 91 & 92 & 93 & 94 & 95 & 96 & 97 & 98 & 99 \\ \text { DISTANCE } & 5942 & 6016 & 6090 & 6164 & 6239 & 6313 & 6387 & 6462 & 6536 & 6610 & 6684 & 6759 & 6833 & 6907 & 6981 & 7056 & 7130 & 7204 & 7278 & 7353\end{array}$

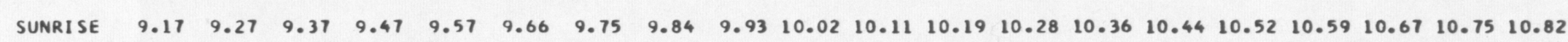
$\begin{array}{lllllllllllllllllllll}\text { SUNSET } & 3.04 & 3.04 & 3.04 & 3.05 & 3.05 & 3.05 & 3.05 & 3.06 & 3.06 & 3.06 & 3.06 & 3.07 & 3.07 & 3.07 & 3.07 & 3.08 & 3.08 & 3.08 & 3.08 & 3.09\end{array}$ $\begin{array}{ll}\text { NUMBER } & 100 \\ \text { DISTANCE } & 7427\end{array}$

SUNRISE 10.89

SUNSE T 3.09 
DIURNAL VARIATION AND PERCENTAGE OF DARKNESS ON NBA-FRANKFURT PATH

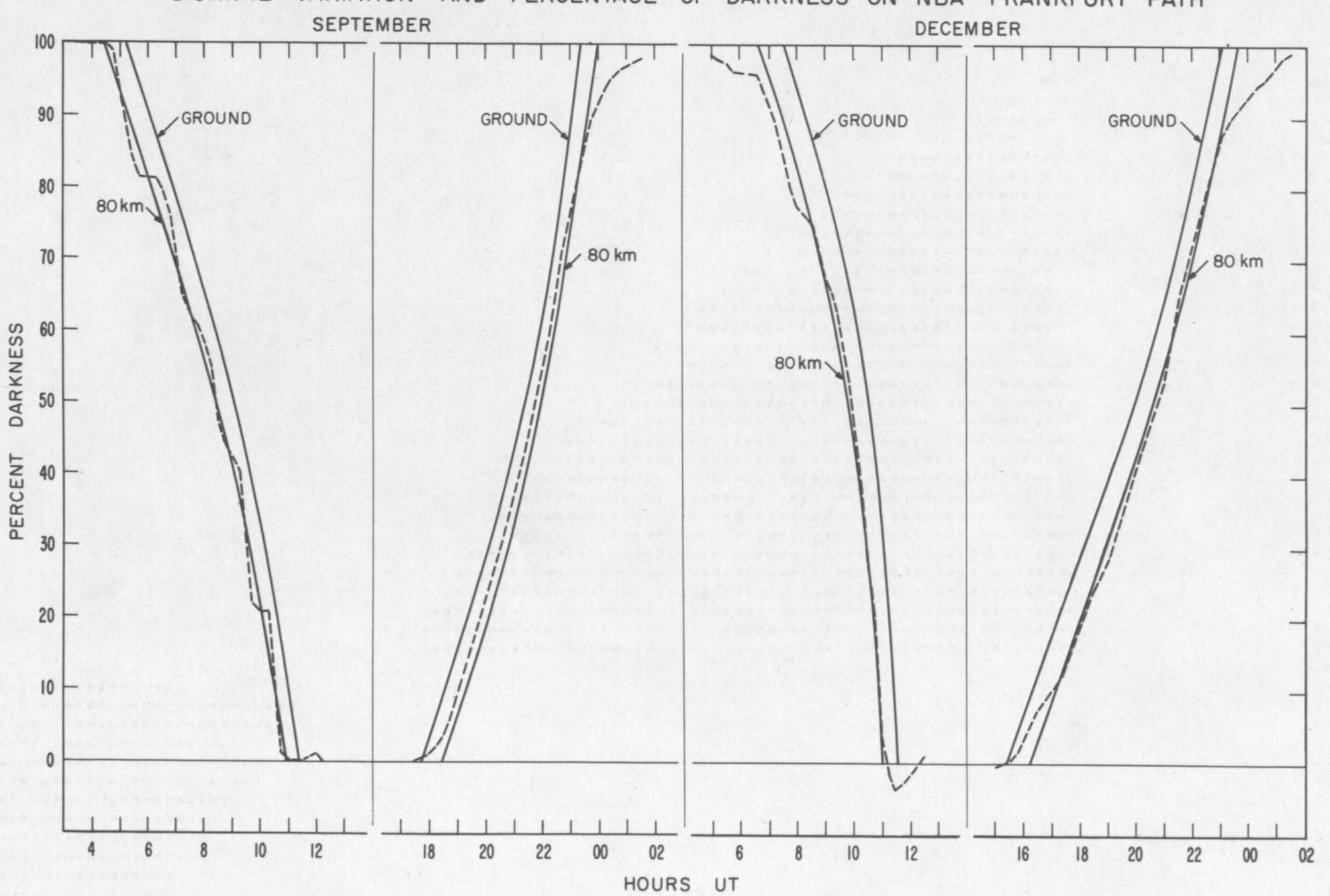

Figure 4. Comparison of phase delay (dashed line) expressed as a percentage of its total diurnal change, with variation of the
amount of path in darkness at $80 \mathrm{~km}$ and ground level (solid lines). NBA (Canal Zone) to Frankfurt. (Screening ht. = $30 \mathrm{~km}$.) 


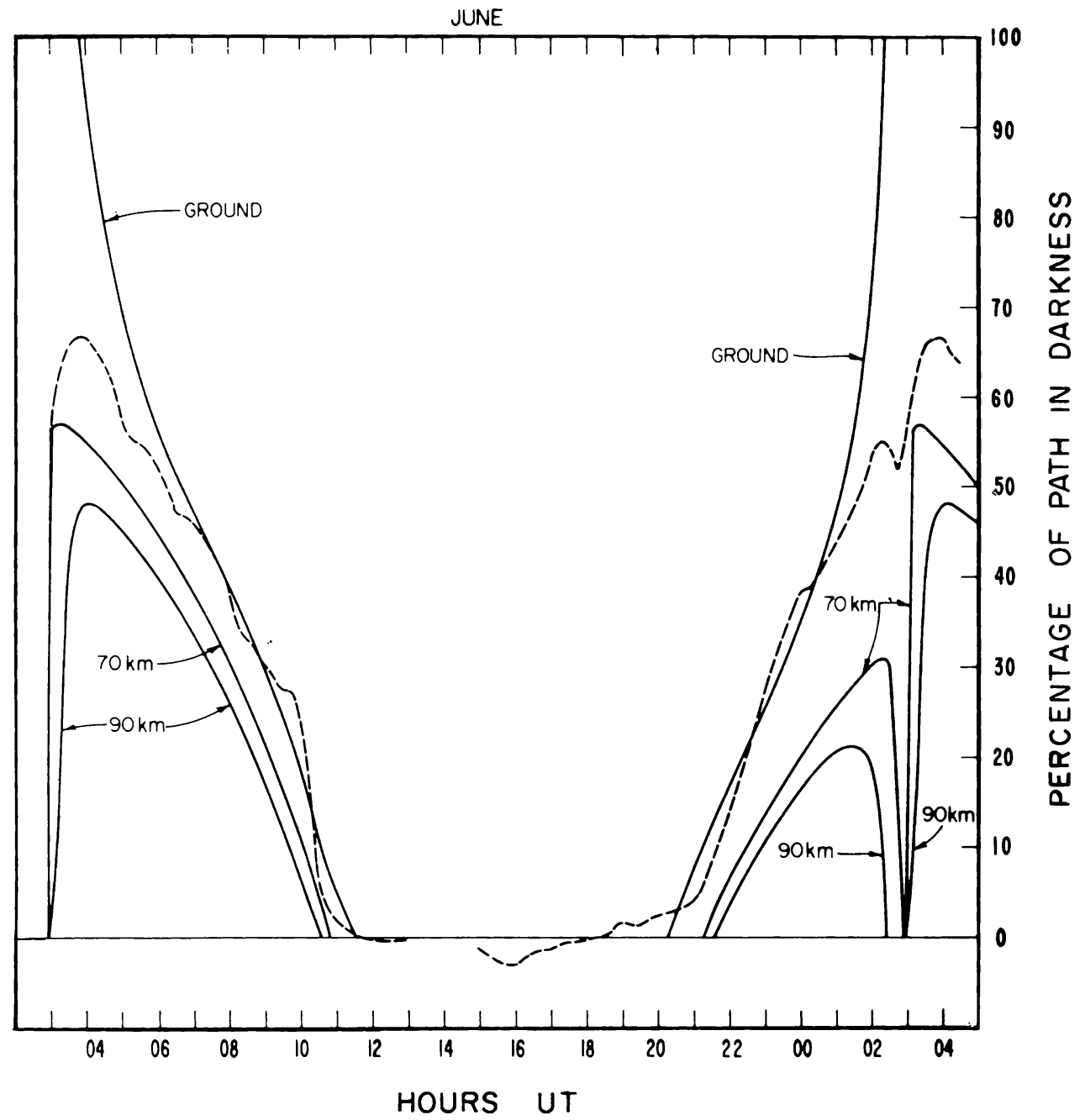

Figure 5. Comparison of phase delay expressed as a percentage of its total diurnal change, with variation of amount of path in darkness at $90 \mathrm{~km}, 70 \mathrm{~km}$, and at ground level (solid lines). (Screening ht. $=30 \mathrm{~km}$.) 


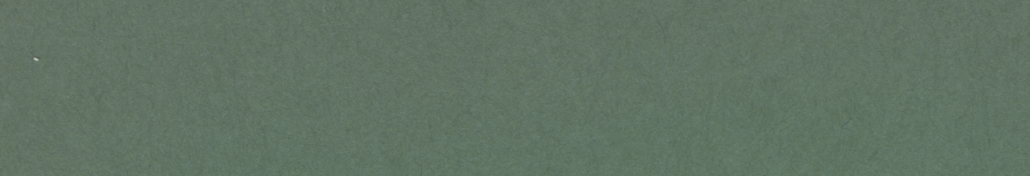

(7)

singe

8

35

3. 
U.S. DEPARTMENT OF COMMERCE WASHINGTON, D.C. 20230

OFFICIAL BUSINESS
POSTAGE AND FEES PAID U.S. DEPARTMENT OF COMMERCE 